\title{
Borderline personality disorder and nursing approach
}

\author{
Marindela Pergjini', Evangelos C. Fradelos', loanna V. Papathanasiou ${ }^{1,3}$ \\ ${ }^{1}$ Nursing Department, University of Thessaly, Larissa, Greece \\ ${ }^{2}$ General Department, University of Thessaly, Larissa, Greece
}

${ }^{3}$ Community Nursing Lab, Nursing Department, University of Thessaly, Greece

\begin{abstract}
Introduction: Borderline Personality Disorder, is one of the ten Personality Disorders. These Disorders are split into three categories, with the Borderline being part of the second one where elements of dramatization and emotional instability are frequently evident.

Purpose: The purpose of the present study is to investigate and highlight the characteristics, the treatment and nursing approach for people with this disorder.

Methodology: The study material consisted of articles on the topic found in Greek and international databases such as: PubMed, Cochrane, Hellenic Academic Libraries Association (HEAL-Link), Scopus and PsycINFO, using keywords as: "Borderline Personality Disorder", "Diagnosis", "Therapy", "Treatment", "Holistic Care", "Nursing Care".

Results: Bordeline Personality Disorder is characterized as a condition in which a person differs significantly from the average of people, about how he thinks, perceives, feels or relates to others. Treatment for this Disorder does not exist, however medication is used to remission the symptoms. Nurses are part of the treatment team. They're going to help the patient learn to live with the symptoms of his disorder. As these people are special patients, nurses must learn from their training not to focus on the patient's problem, but on the patient himself.

Conclusions: The key characteristics of Borderline are impulsivity and instability in interpersonal relationships, self-image and emotions. As there is no treatment, nurses as members of the treatment team must develop a relationship of trust with the patient in order to be able to help him in his recovery. It is important for nurses to be able to properly approach the person with Borderline Personality Disorder to learn to adapt according to his personality
\end{abstract}

\section{Keywords}

Borderline Personality Disorder, Diagnosis, Medication, Holistic Care, Nursing Care.

Address for correspondence:

Marindela Pergjini, RN, Nursing Department, University of Thessaly, Larissa, Greece. e-mail: marindela96@gmail.com

This work is licensed under a Creative Commons Attribution-

NonCommercial 4.0 International License (CC BY-NC 4.0).

\section{(c) (i) (9)}

(C) Copyright: Pergjini, Fradelos, Papathanasiou, 2020

Submitted for publication: 18 June 2020

Received: 18 June 2020

Accepted for publication: 14

Licensee NDSAN (MFC- Coordinator of the NDSAN), Italy

DOI: http://dol.org/10.32437/mhgcj.v3i1.81 


\section{Introduction}

Borderline Personality Disorder, according to the American Psychiatric Association and the DSM is one of the ten Personality Disorders. These Disorders are split into three categories, with the Borderline being part of the second one where elements of dramatization and emotional instability are frequently evident. (APA, 2013). The key characteristics of BPD are impulsivity and instability in interpersonal relationships, self-image and emotions. These symptoms tend to start in the early years of adulthood and affect various circumstances of the patient's life. This Disorder is usually found in the $1 \%-3 \%$ of the general population while clinical patients cover approximately $10 \%$ of the cases. Individuals with Borderline Personality Disorder can also have an intense tendency of suicidal ideation and selfharm. The suicide rate is $8 \%-10 \%$ (Stroud \& Parsons, 2012) but there are few epidemiological data for BPD patients in Greece. In order for the diagnosis of the Personality Disorder to be accurate, the individual needs to be over the age of 18 because its personality is still being shaped under this age. What is more, the patient's behavior should be persistent over time and not transient.

\section{Purpose}

The purpose of the present study is to investigate and highlight the characteristics of Bordeline Disorder as defined by the World Health Organization and the American Psychiatric Association, the treatment consisting, and the nursing approach for people with this disorder.

\section{Methodology}

The study material consisted of articles on the topic found in Greek and international databases such as: PubMed, Cochrane, Hellenic Academic Libraries Association (HEAL-Link), Scopus and PsycINFO, using keywords as: "Borderline Personality Disorder", "Diagnosis", "Therapy", "Treatment", "Holistic Care", "Nursing Care".

\section{Results}

\section{Historical background}

In older times, scientists of the psychodynamic direction used the terms

"Ambulatory schizophrenia" and "Pseudoneurotic schizophrenia" in order to describe a group of people that is in-between neurosis and psychosis, with the main characteristics of instability (emotional, interpersonal relationships and self-image) and impulsivity. In 1938 the term "Borderline" was suggested for the first time by the psychoanalyst Stern, in an effort to describe a patient who was in-between neurosis and psychosis and was mentally unstable and difficult to manage (Eby \& Brown 2010). The Borderline Personality Disorder as an official term, was finally institutionalized in the early 1980's at DSM-III and constitutes one of the ten Personality Disorders since then (Lenzenweger \& Clarkin, 2005).

\section{Clinical Characteristics}

Individuals that have been diagnosed with Borderline Personality Disorder are usually impulsive and unstable in their interpersonal relationships, self-image and emotions. These symptoms appear in the early adult life of the individual. They may appear as responses to personal and social situations and they differ, fundamentally, from the way the average person understands, thinks, feels and relates with others

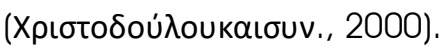

The intense and unstable relationships are these people's main characteristic. They tend to be extremely sensitive to the idea of rejection and the fear of abandonment. When it comes to their interpersonal relationships, they usually have non-realistic expectations and show increased emotional instability in the case of unexpected disappointment and obvious rejection (Dubovsky \& Kiefer, 2014; Andrew et al., 201 1). Borderline Personality Disorder is, also, characterized by an unstable self-image and self-worth. The life goals, plans, values, sexual identity and friends of the BPD patient may change in an abrupt way and that is why these people respond better to a predictable and structured environment (Eby \& Brown, 2010).

Moreover, impulsivity is one more key element of the BPD. The diagnosis requires impulsivity in at least two sections that could be proven selfdestructive, such as gambling, irresponsible waste of money, reckless driving, bulimic eating, unprotected sex, substance abuse or selfharming behavior (Giannouli et al., 2009; Nehls, 2000). This type of impulsivity differs from the impulsive behavior that appears during the manic episodes where it is prolonged and accompanied by other symptoms of mania such as grandeur, stressful speech and lack of need for sleep.

Self-harming is, also, frequent in this Disorder. More specifically, $75 \%$ of the individuals that have been diagnosed with Personality Disorder and an even higher percentage of the clinical patients have attempted self-harm (Geoffrey et al., 2016). An $8-10 \%$ of the patients have successfully attempted suicide (Tomko et al., 2014) but there are numerous cases of self-harm without suicide, such as self-cutting, scratches or 
burns on themselves which are much more common.

The aforementioned behavior, often, occurs when the person is worried about possible abandonment or rejection. It can also be seen during episodes of de-realization or depersonalization (Bach \& Sellbom, 2016). Depersonalization consists of a detachment within the self, in which the individual feels like he is placed outside of his body and looks at himself from a distance. He doesn't feel pain if he is injured. Some patients claim that the pain from cutting and burning reminds them that they're alive (Eby \& Brown, 2010).

\section{Diagnosis}

The immense need for a "common language" in clinical psychiatry, not only on an international level but also on a national, brought to the foreground the formation of the two taxonomic systems:

1) the Diagnostic and Statistical Manual of Mental Disorders (DSM), the American Psychiatric Association and

2) the International Classification of Diseases (ICD) of the World Health Organization.

These two systems are the tools for the classification and the diagnosis of mental illnesses and use specific diagnostic criteria in order for those illnesses to be diagnosed, including the Borderline Personality Disorder.

The fifth edition of the Diagnostic and Statistical Manual of Mental Disorders (DSM-5) includes nine diagnostic criteria. The individuals with Borderline Personality Disorder should have at least five of these criteria which should be evident in various circumstances of the person's life (APA, 2013).

Borderline Disorder patients make excessive efforts to prevent either an existing or an imaginary rejection. When they get the feeling of abandonment, they may intentionally change their self-image and behavior. They, also, experience intense anger even when separation is inevitable. They, often, associate abandonment with the belief that they are "bad" themselves. The intense efforts to avoid abandonment may lead to impulsive actions, like this of self-harm (Bach \& Sellbom, 2016).

Furthermore, the unstable and intense interpersonal relationships are a behavioral characteristic of Borderline Disorder patients (Sellbom et al., 2014). They tend to idealize a friend, partner or mental assistant from their very first contact, to spend time together and share details of their personal life. However, they can easily change this sympathy into devaluation. The feel that this person does not give them time or listen to them, and mainly does not show his support.

The main feature of this Disorder, is impulsive behavior in at least two sections that could be proven catastrophic, such as excessive food or alcohol consumption, substance abuse, dangerous sexual intercourse and reckless driving (APA, 2013).

There may be an identity crisis with an intense and unstable self-image (Trull et al., 2011). The individual feels the need to drastically and dramatically change its self-image which is characterized by a shift in goals, values and professional aspirations. There may be a sudden change in his views and plans about his career, sexual identity and values (Tomko et al., 2014). Such behaviors, occur in situations in which the individual feels the lack of a meaningful relationship, progress and support.

Borderline Disorder patients adopt a continuous suicidal behavior or the habit of selfharm (APA, 2013). The repeated suicide attempts are often the reason why these people seek for help. These acts are overshadowed by threats of separation or rejection. Self-harm can occur during fun experiences and often brings relief to the individual.

Another criterion is the emotional instability which is caused by intense episodic discomfort or anxiety and lasts for a couple of hours or, very rarely, for a few days (Trull et al., 2011). The discomfort of people with Borderline Disorder is often disturbed during periods of anger, panic or despair and rarely by periods of calmness or satisfaction.

These patients are, also, characterized by a chronic feeling of emptiness (APA, 2013). They can easily get bored of anything and are constantly looking for something to do. They have a difficulty in controlling their anger which is usually inappropriate. This anger takes place when they feel neglected, embarrassed or abandoned.

In periods of intense stress, individuals with Borderline Disorder experience transient paranoid ideationor severe detachment symptoms [APA, 2013]. These episodes tend to be transient and they usually last a few minutes, hours and, very rarely, days. They occur during periods of realistic or imaginary abandonment and usually go away when another person's stay is perceived (Trull et al., 2011).

On the other hand, the World Health Organization, through the International Classification of Diseases (ICD-10), suggests that there should be a separate description of five areas - characteristics for the recognition of Personality Disorder. According to ICD-10, the individual should exhibit three of the general 
characteristics of Personality Disorders and at least two of the characteristics of Borderline

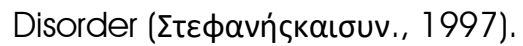

According to the basic criteria of the ICD-10, at first, there should be an indication of the patient's characteristics and the permanent patterns of the mental experience and behavior, as a whole, should be significantly deviant from the culturally expected and accepted rules. Such a deviation must be manifested in more than one area, like cognitive function, emotionality, control over impulses and satisfaction of needs, and how the individual relates to others and handles interpersonal situations. This deviation should be present extensively as a behavior that is inelastic, maladaptive or dysfunctional across a wide range of personal and social situations. What is more, there should be mental stress or an unpleasant impact on the social environment, or both. There must be an indication that the deviation is stable and long-lasting and has begun in late childhood or adolescence. Deviation cannot be interpreted as a manifestation or consequence of other mental disorders, and at the same time an organic brain disease, trauma or dysfunction must be excluded

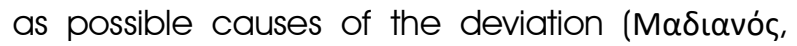
2006).

Provided that there will be a recognition of at least three criteria of the general diagnostic criteria, the ICD-10 suggests the following diagnostic criteria for Borderline Disorder. It is mainly characterized by disturbances and uncertainty about oneself, goals and inner preferences. Patients usually have a predisposition to engage in intense and unstable relationships that often lead to emotional crises (Whewell et al., 2000). They are filled with a sense of grandeur and become very manipulative with people around them. As soon as they realize that those around them do not embrace their grandeur, they will become quite hostile. These people go to great lengths to avoid abandonment. In this endeavor, they display repeated threats or acts of self-harm. Chronic feelings of emptiness, as well as negative emotions and bad mood are distinctive

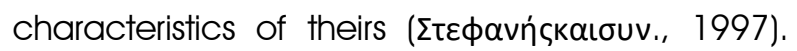
Individuals with Borderline Disorder show severe emotional instability, and they are also very anxious and usually depressed. They are, also, detached andcarry a pattern of indifference. Apart from that, they do not feel the need to develop intimacy with others and are mostly

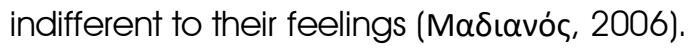

The symptoms should be intense to make the diagnosis and associated with weakened psychosocial function. Once the diagnosis of Borderline Personality Disorder is made, the proposed ICD-10 model includes the assessment of the condition of individuals with BPD as mild,

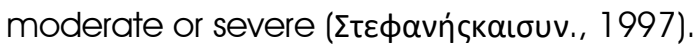

Looking at the two taxonomic systems DSM-5 and ICD-10, there do not seem to be major differences in the recognition of Borderline Disorder, however some differences are noticeable. One of them is the way of diagnosing Borderline Disorder, in DSM-5 there are nine diagnostic criteria for the recognition of Borderline Disorder, while in ICD-10 the diagnosis of Personality Disorder comes first and then the recognition of Borderline Disorder. Also, the absence of psychosis is observed as a diagnostic criterion in ICD-10. The element of psychosis, according to ICD-10, seems to be a diagnostic criterion of schizophrenia and schizotypal personality disorder and not of Borderline Disorder.

Despite the already existing diagnostic criteria, further research seems to be needed to determine whether some criteria should be more important than others. For instance, self-harm and suicide attempts along with unstable interpersonal relationships may be more important signs in the diagnosis of Borderline Personality Disorder.

\section{Therapy/Treatment}

Ensuring the right treatment for people with Borderline Personality Disorder is especially important. The initial priority in the psychosocial treatment of Borderline Disorder is to avoid selfharm. Other issues that need attention are mood problems and the impulsive behavior. The treatment of this Disorder is long lasting. It requires the individual to work with a team of health professionals.

Dialectical Behavioral Therapy is a cognitive and behavioral psychotherapy used specifically for Borderline Personality Disorder. Dialectical Behavioral Therapy has been shown to reduce suicidal behavior and the need for hospitalization, while increasing interpersonal functionality and anger control (Beck et al., 2015).

Equally important is psychopharmacology, which should be taken as complementary therapy and not as the main. Polypharmacy is a common problem faced by people with Borderline Disorder. Research shows that $80 \%$ of people diagnosed with Borderline Disorder are on medication, while $40 \%$ of them seem to be receiving more than 3 medications (Gunderson \& Berkowitz, 2003). Serotonin reuptake inhibitors are the most widely used class of drugs in people with Borderline Disorder that help with the symptoms of depression (Zanarini et al., 2001). Another class of drugs used is that of 
benzodiazepines. Although there is no research data to support their use in people with Borderline Disorder, they are used to treat anxiety and emotional instability. However, they should be prescribed, if necessary, very carefully and in small doses (Stoffers \& Lieb, 2015; Zanarini et al., 2001). Anticonvulsants seem to have positive effects on reducing depression and interpersonal problems.Another widely used class of drugs are antipsychotics, which are used both for psychotic symptoms and to manage mood instability (Lieb et al., 2010).

All classes of drugs should be prescribed with great caution as there are individuals with Borderline Disorder who are prone to suicide attempts, with the result that the medication can be fatal in case of overdose. Due to the different classes of medications available, medication should be adjusted according to the symptoms of the person with Borderline Disorder and unnecessary medication should be avoided.

\section{Nursing Approach}

The nurse is an integral part of the team of health professionals in which he comes in contact with people with Borderline Disorder. Peplau is the one who stressed the importance of the relationship that is being developed between the nurse and the patient. She, also, pointed out how important it is for the patient (as a human) to be the focal point of nursing care and not his problem.

Peplau's model, which is based on psychodynamic and psychodramatic theories, seems to be the most appropriate for the nurses' proper treatment of people with Borderline Disorder. Peplau described 4 interrelated and overlapping phases in the nurse-patient relationship [Peplau, 1997]. The orientation phase in which the nurse understands and evaluates why the person is at the point of receiving help. Then, follow the phases of identification and exploitation, which are phases of work, patient support in recognizing internal dissonance as well as developing and testing strategies that reduce internal dissonance. Finally, there is the analysis phase, where the patient uses these strategies to reduce and resolve internal dissonance and psychological pain [Peplau, 1992].

The role of the nurse in the treatment of people with Borderline Disorder is mainly supportive. The nurse, is the one who will help these people learn to cope with the demands of daily life and to meet their basic needs [O'Connell \& Dowling, 2013]. This procedure should be done in collaboration with the patient, so it is especially important to develop a trust relationship. The nurse should encourage the person to make small gradual decisions about his daily life, so the person takes on a role in the treatment team by helping in his smoother and faster recovery and well-being.

People with Borderline Disorder are people who have difficulty in developing interpersonal relationships so they need to be given more time to develop a proper therapeutic relationship. This proper relationship, allows the nurse to determine the roles he will adopt as well as the nursing interventions he will follow and the roles the person must adopt in order to proceed with the recovery (Papathanasiou et al., 2013; Stockmann, 2005).

The nurse is responsible for a big part of the patient's care. In order to be able to provide proper nursing care and for his patients to have the right healing process it would be right to try to see the person and not the problem. Unfortunately, the nurse during his training is detuned in his attempt to learn the practical part correctly, forgetting that he must also understand the psychosynthesis of his patient (Henderson, 2002).

\section{Conclusions}

People with Borderline Personality Disorder are characterized by a rather peculiar behavior, with impulsivity, tendencies of self-injury and unstable interpersonal relationships being key features. The diagnosis of the Disorder cannot be made before the age of 18 , as until then the personality is still being formed. However, it is important to diagnose Borderline Disorder early so that people can learn how to control their impulsivity and reduce the tendency for self-harm. Nurses have an important role in this process, as they are next to these people teaching them how to cope with meeting their basic needs. In order to do this, a therapeutic relationship must be developed between nurses and patients, but individuals with Borderline Disorder find it difficult to develop and maintain a relationship. Knowledge of the salient features is essential to create a favorable therapeutic alliance, to increase the patient's selfawareness, to plan realistic therapeutic goals and to match the treatment with the individual's personality. Nursing has a human-centered character so nurses should not forget that behind the problem they face, there is a person who is the center of attention.

\section{Conflict of interest}

Authors declare that they have no conflict of interests.

\section{References}


American Psychiatric Association. (2013). Diagnostic and Statistical Manual Of Mental Disorders. 5th ed. Washington: American Psychiatric Association.

Andrew, E. et al. (2011). Personality Disorder Types Proposed for DSM-5. Journal of Personality Disorders, 25(2), 136-169. Accessed From April, 2011, Available From: https://doi.org/10.1521/pedi.2011.25.2.136 .

Bach, B. \& Sellbom, M. (2016). Continuity between DSM-5 Categorical Criteria and Traits Criteria for Borderline Personality Disorder. SAGE Journals, 61(8), 489-494. Accessed From 18th March, 2016, Available From: https://doi.org/10.1177/0706743716640756.

Beck, A., Davis, D. \& Freeman, A. (2015). Cognitive Therapy of Personality Disorders. 3rd ed. New York: The Guilford Press.

Brieger, P. et al. (2000). The Relationship Between Five-Factor Personality Measurements and ICD-10 Personality Disorder Dimensions: Results from a Sample of 229 Subjects. Journal of Personality Disorders, 14(3), 282-290. Available From:

https://doi.org/10.1521/pedi.2000.14.3.282.

Dubovsky, A. \& Kiefer, M. (2014). Borderline Personality Disorder in the Primary Care Setting. Medical Clinics of North America, 98(5), 10491064. Accessed From September, 2014, Available From: https://doi.org/10.1016/j.mcna.2014.06.005.

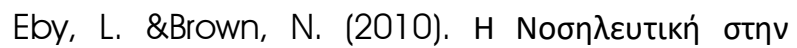

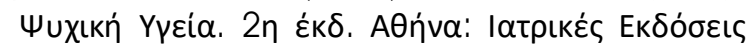

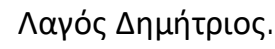

Geoffrey, L. et al. (2016). Mental health nurses' attitudes, behaviour, experience and knowledge regarding adults with a diagnosis of borderline personality disorder: systematic, integrative literature review. Journal of Clinical Nursing, 25(13-14), 1848-1845. Accessed From 3th May, 2016, Available From: https://doi.org/10.1 11 1/jocn. 13202.

Giannouli, H. et al. (2009). Attitudes, knowledge and experience of nurses working in psychiatric hospitals in Greece, regarding borderline personality disorder: a comparative study. Journal of Psychiatric and Mental Health Nursing, 16(5), 481-487. Accessed From 7th May, 2009, Available From: https://doi.org/10.1 111 /j.1365-

2850.2009.01406.x.
Gunderson, J. \& Berkowitz, C. (2003). An Introduction To Borderline Personality Disorder. Diagnosis, Origins, Course, and Treatment. New York: NEPDA.

Henderson, S. (2002). Factors impacting on nurses' transference of theoretical knowledge of holistic care into clinical practice. Nurse Education in Practice, 2(4), 244-250. Accessed 22th April, 2002, Available From: DOI: 10.1016/s14715953(02)00020-3.

Lenzenweger, F. \& Clarkin, J. (2005). Major Theories of Personality Disorder. 2nd ed. New York: The Guilford Press.

Lieb, K. et al. (2010). Pharmacotherapy for borderline personality disorder: Cochrane systematic review of randomised trials. Cambridge University Press, 196(1), 4-12. Accessed From 2 January, 2018, Available From: doi: 10.1 192/bjp.bp. 108.062984.

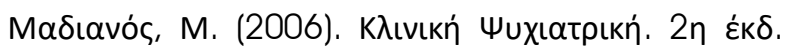

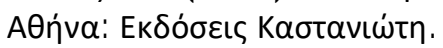

Nehls, N. (2000). Being a case manager for persons with borderline personality disorder: perspectives of community mental health center clinicians. Archives of Psychiatric Nursing, 14(1), 12-18. Accessed From February, 2000, Available From: DOI: 10.1016/s0883-9417(00)80004-7.

O'Connell, \& Dowling, M. (2013). Community psychiatric nurses' experiences of caring for clients with borderline personality disorder. Mental Health Practice, 17(4):27-33. Accessed From 1st March, 1013, Available From:

https://dx.doi.org/10.7748/mhp2013.12.17.4.2 7.e845.

Papathanasiou, I., Slavou, M. \& Kourkouta, L. (2013). Holistic Nursing Care: Theories and Perspectives. American Journal of Nursing Science, 2(1), 1-5. Accessed From 20th February, 2013, Available From: doi: 10.11648/j.ajns.20130201.11.

Peplau, HE. (1997). Peplau's theory of interpersonal relations. SAGE Journals, 10(4), 162-167. Accessed From 1 October, 1997, Available From: DOl: $10.1177 / 089431849701000407$.

Peplau, HE. (1992). Interpersonal relations: a theoretical framework for application in 
nursing practice. SAGE Journals, 5(1), 13-18. Accessed From 1 January, 1992, Available From: DOl:10.1177/089431849200500106.

Sellbom, M. et al. (2014). Convergence between DSM-5 Section II and Section III diagnostic criteria for borderline personality disorder. SAGE Journals, 48(4), 325-332. Accessed From 19th November, 2014, Available From: https://doi.org/10.1177/0004867413511997.

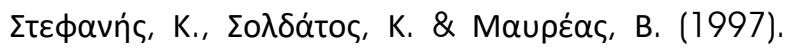

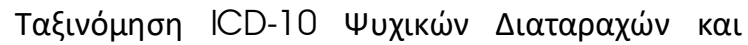

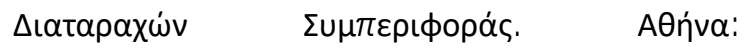

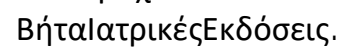

Stockmann, C. (2005). A literature review of the progress of the psychiatric nurse-patient relationship as described by Peplau. Issues in Mental Health Nursing, 25(9), 911-919. Accessed 9th July, 2005, Available From: https://doi.org/10.1080/01612840500248197.

Stoffers, J. \& Lieb, K. (2015). Pharmacotherapy for Borderline Personality Disorder-Current Evidence and Recent Trends. Current Psychiatry Reports. 17(534). Accessed From 21 November, 2015, Available From: https://doi.org/10.1007/s1 1920-014-0534-0.

Stroud, J. \& Parsons, R. (2012). Working with borderline personality disorder: A small-scale qualitative investigation into community psychiatric nurses' constructs of borderline personality disorder. Personality and Mental
Health, 7(3),242-253. Accessed From 23th August, 2012, Available From: https://doi.org/10.1002/pmh. 1214.

Tomko, R. et al. (2014). Characteristics of borderline personality disorder in a community sample: comorbidity, treatment utilization, and general functioning. Journal of Personality Disorders, 28(5), 734-750. Accessed From October, 2014, Available From: doi: 10.1521/pedi_2012_26_093.

Trull, T. et al. (2011). DSM-5 Borderline Personality Disorder: At the Border Between a Dimensional and a Categorical View. Curr Psychiatric, 13, 43-49. Accessed From 24th November, 2011, Available From: https://doi.org/10. 1007/s1 1920-010-0170-2.

Whewell, P. et al. (2000). Does the ICD 10 classification accurately describe subtypes of borderline personality disorder?. British Journal Of Medical Psycology, 73(4), 483-494. Accessed From 14th February, 2000, Available From:

https://doi.org/10.1348/000711200160679.

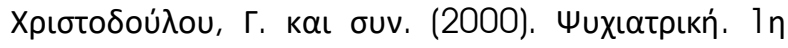

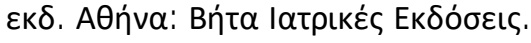

Zanarini, M. et al. (2001). Treatment histories of borderline inpatients. Comprehensive Psychiatry, 42(2), 144-150. Accessed From March, 2001, Available From: https://doi.org/10.1053/comp.2001.19749 